\title{
Lidil
}

Revue de linguistique et de didactique des langues

\section{Expressions vocales et traces verbales de l'émotion dans l'entretien de conseil en apprentissage des langues}

Emmanuelle Carette, Carlos Meléndez Quero et Éric Thiébaut

\section{OpenEdition}

\section{Journals}

Édition électronique

URL : http://journals.openedition.org/lidil/3327

DOI : 10.4000/lidil.3327

ISSN : 1960-6052

Éditeur

UGA Éditions/Université Grenoble Alpes

\section{Édition imprimée}

Date de publication : 1 novembre 2013

Pagination : 171-187

ISBN : 978-2-84310-260-8

ISSN : $1146-6480$

Référence électronique

Emmanuelle Carette, Carlos Meléndez Quero et Éric Thiébaut, «Expressions vocales et traces verbales de l'émotion dans l'entretien de conseil en apprentissage des langues », Lidil [En ligne], 48 | 2013, mis en ligne le 01 mai 2015, consulté le 01 mai 2019. URL : http://journals.openedition.org/ lidil/3327 ; DOI : 10.4000/lidil.3327 


\title{
Expressions vocales et traces verbales de l'émotion dans l'entretien de conseil en apprentissage des langues
}

\author{
Emmanuelle Carette, Carlos Meléndez Quero* \\ et Éric Thiébaut**
}

\begin{abstract}
Résumé
La recherche concernant la représentation verbale d'émotions s'est essentiellement intéressée à l'inventaire, la description, la classification du lexique des émotions, réduisant l'étude aux possibilités sémantiques et conscientes du phénomène. Par ailleurs, la nature et l'impact des états émotionnels sur l'apprentissage autodirigé des langues restent largement méconnus. Un premier objectif de cette étude est ainsi d'identifier des émotions dans l'entretien de conseil, sur la base du ton de la voix. Un deuxième objectif est d'identifier des traces verbales qui sont liées aux émotions négatives. Le statut et l'utilisation de ces traces verbales sont discutés.
\end{abstract}

\begin{abstract}
Research on the verbal representation of emotions has so far focused on listing, describing and classifying the vocabulary of emotions, thus limiting its focus to semantic possibilities and conscious phenomena. Further, in the specific context of learner counseling, the nature and impact of emotional states on self-directed language learning remain largely unexplored. A primary objective of this study is to identify emotions on the basis of tone of voice. A second objective is to identify recurrent or salient verbal traces that can be related to negative emotions. Finally, the status and the potential use of these verbal traces are discussed.
\end{abstract}

* Université de Lorraine, CNRS, ATILF, UMR 7118, équipe Didactique des langues et sociolinguistique (Crapel), Nancy (France).

** Université de Lorraine, INTERPSY EA 4432, Nancy (France). 
Les émotions dans l'apprentissage et l'acquisition font l'objet, depuis deux décennies, d'un intérêt grandissant en didactique des langues. $\mathrm{Si}$ elles sont reconnues comme jouant un rôle important dans l'acquisition de langue étrangère (désormais $\mathrm{LE}^{1}$ ), leur nature et leur rôle dans le domaine de l'apprentissage des langues par enseignement ne font l'objet d'études que depuis une dizaine d'années, les émotions positives en tant qu'éléments de la motivation, les négatives comme facteurs de démotivation (Dörnyei, 2005; Bown \& White, 2011; Méndez López, 2011). Concernant le contexte de l'apprentissage de LE en autonomie, l'étude des émotions n'en est qu'à ses débuts (Hurd, 2008).

Les recherches recensées portent parfois indifféremment sur les affects, les émotions, les sentiments, les humeurs, groupés sous le terme de «variables affectives» (Scherer, 2005; Hurd, 2008; Dewaele, 2011). Il semble nécessaire de distinguer entre ces différents phénomènes. Après avoir présenté le contexte de notre étude et défini notre objet, nous nous proposons de répondre à deux questions : premièrement, quelle est la palette des émotions que l'on peut repérer dans les entretiens de conseil? Deuxièmement, existe-t-il des traces verbales liées à ces émotions ${ }^{2}$ ?

\section{Le contexte de l'étude}

\subsection{L'entretien de conseil}

Les concepts développés par l'équipe du CRAPEL ${ }^{3}$ depuis les années 1970 autour de l'autonomie d'apprentissage et de l'apprentissage autodirigé (Abe, Henner Stanchina \& Smith 1975; Holec, 1990; Riley \& Gremmo, 1997) sont à la base d'un projet de valorisation de la recherche qui a permis de développer un centre privé de formation à l'anglais à visée autonomisante (Carette, Guély \& Pereiro, 2011). On propose aux apprenants de développer leur capacité d'apprendre grâce à un dispositif qui comporte, entre autres, des moments de rencontre et de discussion avec un conseiller : les entretiens de conseil. Ils sont l'occasion d'une interaction à visée pédagogique particulière, qui porte sur

1. Nous ne distinguons pas dans cette étude LE et langue seconde.

2. Pour cet article, seules les traces verbales liées aux émotions à valence négative sont présentées.

3. Cf. textes publiés dans les Mélanges Crapel: <www.atilf.fr/spip.php? rubrique217>. 
les choix que doit faire l'apprenant quant à ses objectifs d'apprentissage de LE, les ressources et activités d'apprentissage, la gestion de son travail (rythme, durée, lieu) et l'évaluation de ses compétences langagières. L'apprenant est aussi amené à évaluer la façon dont il mène son apprentissage. Ainsi, au fil des entretiens, l'apprenant acquiert des capacités métacognitives qui lui permettent de prendre en charge son apprentissage. L'action du conseiller dans ce cadre consiste à écouter ce que lui dit l'apprenant sur lui-même, sur ses expériences d'apprentissage, à ancrer des apports d'information sur ce qu'est une langue, ce qu'est apprendre une langue et donner des conseils méthodologiques (Gremmo, 1995; Carette \& Castillo, 2004; Ciekanski, 2005). Il réagit donc autant aux connaissances de l'apprenant qu'à ses dispositions, humeur, tempérament, sentiments et émotions.

Les contenus cognitifs de ces entretiens de conseil ont été largement décrits dans la littérature sur l'autonomie dans l'apprentissage des langues (Holec, 1990; Gremmo \& Holec, 1987; Riley, 1994; Ciekanski, 2007). Plus récemment, un ouvrage récapitulant les centres d'intérêt des recherches sur l'autonomie (Kjisik, Voller, Aoki \& Nakata, 2009) s'intéresse à plusieurs paradigmes : l'identité des apprenants, leur formation et celle des enseignants, les contextes sociaux de l'autonomie. Mais le jeu des émotions qui se manifestent dans ces entretiens reste encore très largement ignoré. C'est l'objet de notre recherche, et, même si nous nommons les acteurs de l'entretien de conseil d'après leurs rôles sociaux, conseiller et apprenant, ce qui nous intéresse est le fait que ce sont avant tout deux personnes, et qu'une relation se développe entre les deux lors des rencontres.

\subsection{Les entretiens étudiés}

Les entretiens de conseil ont été enregistrés avec du matériel audio et transcrits avec Transcriber ${ }^{4}$. Pour cette étude, nous avons travaillé sur deux séries de cinq entretiens entre conseillers et apprenants adultes: la première série se compose de trois entretiens entre une conseillère femme ( $\mathrm{C} 1,30-35$ ans) et un apprenant adulte (A1, homme, 45-50 ans), et de deux entretiens entre ce même apprenant et une autre conseillère (C2, femme, 30-35 ans); la seconde série inclut cinq entretiens entre C2 et un autre apprenant (A2, homme, 35-40 ans). L'ensemble du corpus

4. Logiciel gratuit de transcription et annotation de corpus. 
étudié représente cinq heures dix minutes, et six mille deux cents tours de parole.

\section{Quelles émotions dans ce contexte?}

\subsection{Définition de notre objet : les émotions}

Un point de vue classique en psychologie considère l'émotion comme une entité à multiples composants. Les plus généraux concernent les réponses neurophysiologiques (sueur, accélération cardiaque), les comportements expressifs (voix, visage, gestes), les expériences subjectives (sentiments) et les tendances à l'action. Notre conception en arrière-plan est fonctionnaliste : les émotions sont considérées comme des réponses à des problèmes adaptatifs dont le plus général est la coordination d'une multitude de modules mentaux (Cosmides \& Tobby, 2000) gouvernant la perception, l'attention, les inférences, l'apprentissage, la mémoire, le choix de buts, etc. Ce statut attribué aux émotions rend intelligibles les relations fréquemment rapportées dans la littérature entre des variables ou des composants cognitifs et émotionnels. Les émotions constituent une réponse, qui peut être automatique, aux situations. Les expressions émotionnelles informent sur la représentation subjective de la situation.

\subsection{Méthodologie : le dispositif de codage des expressions émotionnelles}

Nous avons opté pour la discrimination humaine des émotions. La littérature à propos de la détection automatique des expressions émotionnelles par l'analyse des propriétés du signal sonore fait état de résultats intéressants (par exemple : Cohen, Minor, Najolia \& Hong, 2009; Cowie, Douglas-Cowie, Savvidou, McMahon, Sawey \& Schröeder, 2000), mais montre que le degré de sensibilité discriminative des dispositifs automatiques reste inférieur au degré de sensibilité humaine. Or, la relative discrétion des expressions émotionnelles dans le contexte social des entretiens de conseil exige de recourir à une technique de haute sensibilité.

La discrimination est opérationnalisée par un codage des expressions vocales selon un petit nombre de catégories. Les juges ont pour instruction de négliger le contenu verbal. On ne peut toutefois pas exclure qu'il intervienne dans leur appréhension des expressions émotionnelles. 
On peut questionner la validité de la discrimination humaine. Une revue de Scherer (1989) indique, sur la base d'une trentaine d'études, que des individus sont capables d'inférer des états affectifs à partir d'échantillons vocaux avec un pourcentage d'accord inter-juges moyen de $60 \%$. Une valeur similaire est proposée pour des données interculturelles (Scherer, Banse \& Wallbott, 2001). Ainsi, on postule pour notre étude que la détection d'expressions émotionnelles par un juge qui ne participe pas aux interactions est une approximation acceptable de ce qui peut être détecté par les personnes engagées dans l'interaction.

Les émotions de base au sens d'Ekman (1999) (joie, tristesse, peur, dégout, colère, surprise), qui font l'objet de nombreuses recherches sur les émotions, ne nous semblent pas couvrir la gamme de celles qui se produisent en entretien de conseil. C'est pourquoi nous avons utilisé les résultats des travaux de Carney et Colvin (2010). En se fondant sur les données d'un codage du contenu d'enregistrements vidéo d'interactions dyadiques (79 participants placés dans quatre situations différentes), ils montrent une organisation circulaire des proximités psychologiques entre les catégories d'émotions, ce qui réplique un modèle antérieur de description des émotions (Russell, 1980). Cette structure circulaire est classiquement résumée à l'aide de deux dimensions : l'une, la valence, concerne la polarité avec le caractère plaisant ou au contraire désagréable de l'émotion, et l'autre, l'activation, désigne un degré d'intensité.

Les premières mises en œuvre du dispositif de codage par catégorie ont montré la nécessité de procéder à quelques remplacements et ajustements des catégories empruntées à l'étude de Carney et Colvin (2010). Notre grille finale se compose de dix-sept catégories dont une catégorie «neutre» qu'il nous a semblé préférable de proposer aux codeurs, comme alternative à une absence de choix de catégorie : en effet, de nombreux tours de parole ne se caractérisent ni positivement, ni négativement. Nous avons procédé par itérations successives, jusqu'à l'obtention d'une grille stable permettant de représenter économiquement et sans perte d'information notable la palette des émotions exprimées dans les entretiens de conseil. L'évolution de la grille de codage s'explique également par les données initiales de fidélité inter-juges, c'est-àdire le degré de convergence entre les jugements de différents codeurs. Dans des situations contrastées d'interactions sociales, l'étude de Carney et Colvin (2010) fait état de fidélité inter-juges représentée par un coefficient $\alpha$ moyen de .64, la valeur 1 dénotant une convergence parfaite. Pour notre étude, la valeur moyenne du coefficient $\alpha$ (.56) est légèrement inférieure à celle proposée par Carney et Colvin (2010). Cette 
valeur assez modeste peut s'expliquer, au moins en partie, par les faibles contrastes entre les émotions exprimées. La catégorie «neutre», ne correspondant pas à une expression émotionnelle, n'est pas incluse dans cette analyse.

\subsection{Résultats}

Le tableau 1 met en évidence les catégories d'expression émotionnelle identifiées, avec les fréquences d'occurrence distinguées selon qu'elles concernent les expressions vocales de l'apprenant ou du conseiller.

Le plus souvent, les occurrences des expressions émotionnelles sont faibles, l'essentiel de la tonalité étant la neutralité. Les exceptions concernent la catégorie «empathique» $(9,80 \%$ des cas chez l'apprenant et $43,68 \%$ chez le conseiller) avec un fort déséquilibre entre l'apprenant et le conseiller ainsi que pour la catégorie «content, à l'aise, satisfait» $(18,49 \%$ chez l'apprenant et $4,01 \%$ chez le conseiller $) . \mathrm{Si}$ globalement les expressions émotionnelles ${ }^{5}$ sont plus fréquentes chez le conseiller ( $45,1 \%$ chez l'apprenant et $55,15 \%$ chez le conseiller), elles sont plus dispersées chez l'apprenant. Un déséquilibre supplémentaire concerne les expressions émotionnelles de valence négative qui sont nettement plus fréquentes chez l'apprenant $(10,05 \%)$ que chez le conseiller $(2,24 \%)$ avec un déséquilibre inverse pour les expressions émotionnelles de valence positive (35,04\% chez l'apprenant et 52,9\% chez le conseiller).

\begin{tabular}{|l|c|c|c|c|}
\hline Catégories d'émotions & \multicolumn{2}{|c|}{ Apprenant } & \multicolumn{2}{c|}{ Conseiller } \\
\hline & Fréquence & $\%$ & Fréquence & $\%$ \\
\hline 1 se sent coupable & 16 & 0,50 & 3,5 & 0,11 \\
\hline 2 déçu, découragé, abattu & 52 & 1,69 & 3,5 & 0,11 \\
\hline 3 agacé, tendu, agressif & 30 & 0,97 & 3,5 & 0,11 \\
\hline 4 craintif, inquiet & 22 & 0,70 & 17 & 0,55 \\
\hline 5 s'apitoie sur lui-même & 14 & 0,45 & 0 & 0,00 \\
\hline 6 ennuyé, gêné & 90 & 2,91 & 23 & 0,75 \\
\hline 7 confus, perplexe & 59 & 1,92 & 12,5 & 0,41 \\
\hline
\end{tabular}

5. Calculées après exclusion de la catégorie «neutre». 


\begin{tabular}{|l|c|c|c|c|}
\hline 8 détaché, réservé, absent & 28 & 0,91 & 6 & 0,20 \\
\hline 9 neutre & 1690 & 54,90 & 1375 & 44,85 \\
\hline 10 surpris & 25 & 0,81 & 18 & 0,59 \\
\hline 11 empathique & 302 & 9,80 & 1339 & 43,68 \\
\hline 12 content, à 1'aise, satisfait & 569 & 18,49 & 123 & 4,01 \\
\hline 13 soulagé & 10 & 0,32 & 1 & 0,03 \\
\hline 14 content de soi & 50 & 1,61 & 6 & 0,20 \\
\hline 15 chaleureux & 6 & 0,19 & 46 & 1,50 \\
\hline 16 motivé, enthousiaste & 68 & 2,21 & 39,5 & 1,29 \\
\hline 17 s'amuse, rit & 50 & 1,61 & 49 & 1,60 \\
\hline Total & 3078 & 100 & 3066 & 100 \\
\hline
\end{tabular}

Tableau 1. - Fréquence et pourcentage des occurrences des catégories d'émotion fondées sur un peu moins de 3100 «tours de parole» par locuteur.

\section{Y a-t-il des traces verbales liées aux expressions vocales de l'émotion?}

\subsection{Méthodologie}

À titre exploratoire, nous nous sommes concentrés sur la liste de huit émotions chargées négativement. En nous servant des codages effectués, nous avons étudié tous les tours de parole marqués par des catégories négatives. Nous avons cherché à la fois des régularités et des traits saillants dans le discours de chaque interlocuteur. Nous avons ainsi tenté de dégager les traces verbales les plus significatives associées à chaque expression émotionnelle et de localiser le(s) moment(s) où chacune des émotions apparait.

\subsection{Résultats}

La catégorie «culpabilité » est surreprésentée pour l'apprenant, qui utilise de façon systématique le «je», et souvent des structures négatives, notamment au passé composé, pour exprimer sa culpabilité quand il parle de la méthodologie de travail (j'ai pas dit,j'ai jamais mis en français, j'ai pas regardé), de son passé d'apprenant (je n'ai jamais parlé 
anglais), ou quand il reconnait ne pas avoir accompli le travail demandé (j'ai pas vérifié les réponses, j'ai peut-être pas regardé tout, je l'ai pas $v u$ ). Outre ces constructions récurrentes, l'apprenant manifeste sa culpabilité lorsqu'il décrit ses activités d'apprentissage linguistique antérieures (j'ai fait de l'anglais d'une façon classique, je faisais des choses grammaticales) et actuelles (je fais de la traduction mot à mot, je construis ma phrase en français, je la traduis en anglais).

La deuxième catégorie analysée («déçu, découragé, abattu») est essentiellement présente chez les apprenants, notamment lors des deux premiers entretiens. Les apprenants manifestent leur abattement et leur incapacité à accomplir différentes tâches langagières. Cela se manifeste très fréquemment par la négation des verbes de connaissance, tels que comprendre (j'ai pas compris, j'arrivais pas à comprendre, je comprends pas forcément, je le comprends pas, je comprenais pas, je capte pas), savoir (je savais pas ce que ça \{voulait/veut\} dire, je sais pas le dire) ou connaitre (je connais pas). De même, lorsqu'ils expliquent leurs difficultés à acquérir la méthodologie de travail proposée par le conseiller, ils utilisent la négation des verbes être (je suis pas à cent pour cent), avoir (je vais pas avoir l'automatisme), arriver (à l'oral on n'arrive pas), pouvoir (je peux pas). La déception de l'apprenant peut se manifester à partir d'adjectifs ou groupes adjectivaux à orientation défavorable (compliqué, très très complexe, extrêmement difficile, limité) ou par la négation d'adjectifs à orientation favorable (pas naturel, pas évident); à plusieurs reprises, cette déception est manifestée par des locutions verbales à connotation négative (j'ai toujours eu du mal, j'ai toujours ramé, j'ai tort, on bute), des adverbes (je suis loin du niveau) et même par certains marqueurs exprimant la résignation (enfin) ou un accord non enthousiaste (bon, ben, ouais).

La catégorie «agacé, tendu, agressif» apparait notamment dans le discours de l'apprenant. Sauf quelques exemples isolés relevés au moment de la définition des objectifs de travail (j'ai plus envie), l'apprenant se montre surtout agacé lors de l'entretien initial au moment de discuter avec le conseiller de la méthodologie et du dispositif proposé pour s'améliorer en langue étrangère. Ainsi, l'un des apprenants ne semble pas très convaincu de la méthodologie et l'exprime avec une certaine agressivité à partir de structures de négation autour de la notion d'utilité (ça va pas être très profitable, ça me sert à rien, je vois pas l'intérêt) ou d'expressions au passé servant à illustrer sa contrariété quant au dispositif recommandé (j'avais compris si vous voulez que, je pensais pas qu'il y avait). De même, l'apprenant peut montrer quelques 
réticences à assumer la nouvelle méthode d'apprentissage, qui exige à son égard une série de conditions préalables (faut être déjà stable à un stade d'autonomie pédagogique, ça suppose une autonomie pédagogique assez forte). L'on remarque que ces traces d'agacement ou de tension avec le conseiller disparaissent au fur et à mesure des entretiens de conseil.

La crainte ou l'inquiétude sont des émotions représentées autant dans le discours du conseiller que dans celui de l'apprenant, notamment en ce qui concerne la méthodologie et les ressources d'apprentissage. Elles émergent d'une situation d'incertitude, à laquelle le conseiller répond par des actes langagiers de vérification et de questionnement. L'emploi de la locution verbale «falloir voir» à plusieurs reprises (il faudrait voir ce qu'on en fait, il va falloir voir ce qu'on en fait de tout ça hein, il va falloir voir ce qu'on va en faire) manifeste l'inquiétude du conseiller par rapport au travail de l'apprenant. Dans le discours du conseiller, on peut corréler des formes interrogatives avec des expressions d'inquiétude quant à la méthodologie de travail suivie (est-ce que le one-to-one a été enregistré, est-ce que vous vous êtes réécouté, est-ce que vous avez relevé d'autres choses, vous avez réussi à faire le lien, qu'est-ce que vous en avez pensé de l'enregistrement). De plus, la crainte du conseiller par rapport à la réussite du dispositif est évidente dans certaines formulations (j'aurais peur si là vous commenciez tout de suite avec l'expert). Du côté de l'apprenant, la catégorie «craintif, inquiet» apparait notamment dans l'entretien initial et semble associée aux expressions de négation concernant l'évaluation du niveau de départ (je suis pas du tout à l'aise, je comprends pas grand chose, j'ai beaucoup de mal à évaluer).

La catégorie «s'apitoie» est représentative du discours de l'apprenant lors de son premier entretien avec le conseiller et est toujours liée à l'explication de ses compétences langagières. Parmi les mots choisis par l'apprenant pour s'apitoyer sur lui-même, nous avons repéré la répétition d'adjectifs et de participes à orientation défavorable (perdu, très frustrant, moche, limité, honteux, bloqué), ainsi que la négation de verbes exprimant ses compétences en anglais (j'ai pas de repères, je maitrise pas tout, je perçois pas, je suis pas bon, je peux pas beaucoup, mon niveau est pas très élevé). L'on observe que l'apprenant ne manifeste pas cette émotion négative dans les interactions qui suivent la deuxième rencontre avec le conseiller.

La catégorie "gêné, ennuyé» est très associée à la discussion sur la méthodologie et les ressources d'apprentissage, même s'il n'y a pas 
toujours de trace verbale pour cette expression émotionnelle. À plusieurs reprises, l'émission vocale hum est émise par l'apprenant quand il éprouve de la gêne à expliciter sa pensée au conseiller; d'autres fois, différents marqueurs (ouais, oui, d'accord, euh, voilà, c'est ça) associés à une intonation non enthousiaste constituent une réponse de l'apprenant aux propositions du conseiller. Dans l'entretien initial, l'apprenant exprime sa gêne par rapport au manque de pratique de la langue par l'emploi du verbe avoir au passé et à la forme négative (j'ai pas eu l'occasion, j'ai pas eu de cours d'anglais), et par le choix dans le discours de verbes à connotation négative (je jalousais, je commençais à ramer). Dans les entretiens suivants, la gêne éprouvée par l'apprenant se manifeste souvent au moment d'évaluer ses résultats après le travail effectué. Outre les constructions habituelles de négation (je sais pas dire, je ne le sais pas, je m'en souviens pas, je l'ai pas dit comme $c ̧$ ), l'apprenant choisit des substantifs, adjectifs et adverbes à polarité négative (difficultés, manque, blocages, faute, mauvaises, mal à l'aise). C'est aussi le cas pour le conseiller, qui semble gêné par certaines ressources défaillantes (pas autocorrectives, très techniques) et par des conditions externes qui peuvent perturber l'apprentissage (pas très pratiques, des petites subtilités d'emménagement). Enfin, lors de la discussion des objectifs de travail, le conseiller peut parfois éprouver de la gêne, mais son rôle professionnel et le respect des principes de politesse qui régissent les interactions verbales (Haverkate, 1994) justifient le recours à des stratégies conversationnelles visant à minimiser ses expressions émotionnelles et le conflit avec l'interlocuteur : ce qui est un petit peu embêtant, ce serait dommage de rien en faire et il s'avère en fait que dans les objectifs que euh on avait définis, où la formule un petit peu, le conditionnel serait et la construction il s'avère que servent à réduire l'impact négatif des paroles du conseiller pour préserver l'image de l'apprenant (Leech, 1983).

La catégorie «confus, perplexe» apparait surtout dans le discours de l'apprenant. Ce dernier montre sa confusion lors de l'entretien initial autour du choix des objectifs d'apprentissage (je sais pas, j'ose pas dire). Au cours des entretiens postérieurs, l'apprenant peut montrer des hésitations quant à la validation des objectifs : à partir de la négation pas vraiment, des adverbes peut-être et oui prononcés sans conviction, et notamment des émissions vocales euh et hum, très utilisées dans le discours de l'apprenant notamment pour retarder le moment de donner une réponse au conseiller. Parfois la perplexité de l'apprenant quant à la méthodologie et aux ressources employées peut être associée aux ques- 
tions directes adressées au conseiller (vous êtes sure?), aux demandes de reformulation (c'est-à-dire?) ou au manque de compréhension de la démarche (je sais pas comment organiser, valider ou je sais pas quoi, comment on fait déjà?). De même, la confusion de l'apprenant peut concerner l'autoévaluation du niveau en anglais (je sais pas euh comment l'expliquer, alors attendez, je sais plus).

Enfin, la catégorie «détaché, réservé, absent» est associée au discours de l'apprenant. Ce n'est pas surprenant dans la mesure où le rôle professionnel du conseiller lors des entretiens est d'établir un climat de confiance et de motiver son interlocuteur. On remarque que les traces de détachement dans les entretiens de conseil ne sont pas que verbales. En effet, mises à part quelques expressions sporadiques de l'apprenant (je m'en fous, c'est évident) servant à exprimer un manque d'enthousiasme ou d'implication personnelle, l'aspect le plus significatif de l'analyse de cette catégorie est la réitération d'émissions vocales inarticulées (hum hum).

\section{Discussion}

L'étude tente de décrire la palette des émotions dans les entretiens de conseil et de repérer les traces verbales qui sont liées aux émotions de valence négative. Concernant l'identification des émotions présentes dans ce contexte, de nombreux tours de parole dans nos données ne font pas apparaitre d'émotions distinguables et sont classés neutres. Le mode émotionnel le plus fréquent est une expression empathique (positive) qui est peu distante en valence et en activation de la neutralité. Cette expression est surreprésentée chez le conseiller, liée de façon plausible au rôle qu'il s'attribue dans l'interaction. Chez l'apprenant, deux modes dominent avec les catégories «empathique » (à l'égard du conseiller) et «content, satisfait, à l'aise» (à l'égard de la situation d'entretien). Outre les expressions d'empathie et de satisfaction, dès lors que la valence positive ou négative des émotions est plus affirmée, les fréquences d'occurrence chutent considérablement pour ne plus guère excéder les $2 \%$. La variance des émotions dans la situation de l'entretien de conseil est ainsi assez réduite, comme cela a pu être observé par ailleurs dans d'autres situations d'interaction sociales naturelles (Forbes-Riley \& Litman, 2004; Lee \& Narayanan, 2005). Un deuxième résultat de notre étude sur l'entretien de conseil est que toutes les émotions n'y sont pas représentées (le dégout, la colère intense, le bouleversement). 
Notre étude exploratoire des émotions à polarité négative dévoile que, dans ce contexte d'entretiens, certaines catégories (gêné, perplexe) sont plus fréquentes que d'autres (exprime de la culpabilité, s'apitoie). L'analyse du corpus permet aussi d'observer la répartition des différentes émotions chez le conseiller et l'apprenant et montre que les émotions négatives sont plus nombreuses dans le discours de ce dernier, à l'exception de l'expression de la crainte, répartie également entre les deux participants.

Il apparait également que les distinctions entre émotions sont parfois très fines (peu contrastées) et qu'il n'y a pas toujours une expression verbale que l'on peut lier spécifiquement à une catégorie d'émotion. En effet, nous avons associé à plusieurs reprises une même trace verbale à des catégories émotionnelles variées : par exemple, la marque de la négation, suivie de verbes tels que savoir (je sais pas, je ne le sais pas, je sais plus, je savais pas), arriver (on n'arrive pas, on n'y arrive pas) ou se souvenir (je m'en souviens pas, je m'en souviens plus) a été liée à l'expression de la déception, de la gêne et de la perplexité. Il semble difficile d'établir un glossaire d'expressions ou de traces verbales qui seraient toujours représentatives d'une émotion particulière. Les expressions d'affirmation (oui, ouais), ainsi que des marqueurs d'approbation (d'accord, $O K$ ) en sont particulièrement illustratives. Ces expressions sont souvent employées dans les interactions conversationnelles pour marquer l'empathie envers l'interlocuteur et peuvent être également émises avec une plus grande intensité pour exprimer la satisfaction ou l'enthousiasme; or, malgré cette polarité positive, nous avons trouvé les expressions oui, $O K$ et d'accord dans des catégories négatives (agacé, détaché). Cette caractéristique montre le caractère polyfonctionnel de certaines unités lexicales qui peuvent être employées à des fins discursives très diverses selon la variété des propriétés sonores de la voix et confirme que le code verbal ne joue pas un rôle central dans l'expression de l'émotion. En effet, les traces identifiées ne sont pas toutes de nature verbale, comme c'est le cas de certaines émissions vocales inarticulées (hum hum), lesquelles ont été associées à différentes catégories (craintif, confus, détaché).

Des travaux tels que ceux de Nygaard et Queen (2008) et Pell, Jaywant, Monetta et Kotz (2011) montrent que, dans une situation expérimentale où l'on manipule la congruence émotionnelle entre les aspects vocaux, lexicaux ou sémantiques, celle-ci facilite le traitement cognitif et la détection des émotions. Cette congruence n'apparait pas dans la situation naturelle d'interaction sociale que nous avons 
observée, ce qui met en question les tentatives de détection associant les indices vocaux et verbaux.

La recherche de traces verbales suggère une fracture entre émotion éprouvée et émotion exprimée par un code verbal. Parmi les possibilités du code verbal, on observe que le corpus considéré pour l'étude comporte des éléments lexicaux qui désignent des états affectifs. Ils sont toutefois peu nombreux. Pour l'apprenant, on note par exemple les éléments de polarité négative suivants (avec leur fréquence) : désagréable (1), froid (5), méchant (3), moche (1), perdu (4), réservé (3), réticent (2), saoule (1), malheureusement (2), dommage (2), drame (1), peur (2), souci (5), craindre (1), effrayer (1), ennuyer (2), honte (4), timide (1) et pour le conseiller: défavorable (1), méchamment (1), pressé (2), tension (1), abandonner (1), effrayant (1), embêter (15), ennuie (3), frustré (4), hésite (12), paniquer (1). L'occurrence de ces éléments ne coïncide pas particulièrement avec celle des émotions détectées sur la base des expressions vocales. Celles-ci sont sous un contrôle intentionnel nettement moins étroit que ce n'est le cas des expressions verbales. Elles demeurent susceptibles d'affecter l'état émotionnel des personnes en interaction, mais par contre, elles ne semblent pas constituer des indices des émotions éprouvées dans une situation sociale.

De façon générale, l'étude met en évidence des traces verbales (par exemple, les structures de négation des verbes de connaissance, tels que savoir ou comprendre) différentes des termes qui appartiennent au lexique des «émotions» ordinairement considéré. Ce point fait émerger une interrogation quant au statut de ces traces. Si l'on ne peut les considérer comme des marqueurs des émotions, ces traces apparaissent plutôt comme des marqueurs des situations d'apprentissage. En effet, notre recherche permet de constater que les expressions émotionnelles chargées négativement telles que la culpabilité, la déception ou l'apitoiement apparaissent notamment dans le discours de l'apprenant et sont essentiellement liées soit au manque de travail effectué (culpabilité), soit à l'autoévaluation des compétences langagières pendant l'entretien initial, où l'apprenant se montre souvent découragé ou abattu et s'apitoie sur lui-même. Par ailleurs, d'autres catégories émotionnelles comme l'agacement ou la crainte sont notamment localisées dans des tours de parole concernant la méthodologie d'apprentissage et les ressources ou supports employés. Sous une même thématique, ces deux expressions émotionnelles sont distribuées selon les rôles : l'agacement est manifesté spécifiquement par l'apprenant lors des premiers entretiens quand il découvre le dispositif innovant alors que la crainte est 
plutôt liée au rôle du conseiller, lorsqu'il s'inquiète de l'appropriation de ses conseils méthodologiques. Le lien entre les traces verbales et les émotions nous semble ainsi médiatisé par les situations. Si les traces verbales apparaissent liées aux émotions, c'est parce qu'elles représentent des caractéristiques des situations telles qu'elles sont vécues subjectivement, ce qui constitue l'origine des émotions que l'on détecte par les expressions vocales.

Pour cette étude, nous n'avons utilisé que le seul support audio, ce qui est susceptible d'avoir deux conséquences : la première est une incidence sur la précision de la détection, la seconde est une restriction sur l'éventail des émotions qui peuvent être repérées. La détection des émotions à partir d'un support vidéo permettrait une amélioration de sa précision pour peu qu'il y ait convergence entre les indices, étant fondée sur des modalités supplémentaires d'expression (gestes, visage). On pourrait craindre que le support audio n'ait limité l'éventail des émotions détectées, même si la littérature indique que les expressions vocales sont suffisantes pour accéder à l'essentiel des états émotionnels. Le support visuel est susceptible de permettre la détection d'une émotion comme le dégout dont la manifestation est médiatisée prioritairement par l'expression du visage plutôt que par la voix. En conséquence, une palette distincte des émotions pourrait être obtenue, ainsi que d'autres traces verbales. Toutefois l'enregistrement vidéo n'est pas sans inconvénients, à savoir la présence de la caméra qui intimide certains apprenants et peut inhiber l'expression de leurs émotions.

\section{Conclusion}

Cette étude est un premier pas dans un projet d'élucidation du jeu et des effets des émotions qui se manifestent dans les entretiens visant l'acquisition de capacités métacognitives par l'apprenant. Elle fournit un système de description des émotions dans cette situation, qui peut permettre aux futurs conseillers de disposer à priori d'une double connaissance, celle des émotions susceptibles d'émerger dans la situation de l'entretien de conseil, et celle de leur dépendance avec les thématiques (objectifs d'apprentissage, ressources, etc.). Ces connaissances pourraient servir à former les futurs conseillers à anticiper l'émergence d'émotions selon les thématiques abordées, et donc à s'y préparer.

L'utilisation de ce système de description a permis d'identifier des traces verbales pour les émotions de valence négative, qui apparaissent davantage comme des marqueurs de situations que d'émotions. Dans 
la mesure où les traces verbales renseignent sur les propriétés subjectives des situations, elles constituent des indices des croyances de la personne à propos des types de problèmes considérés (méthodologie, autoévaluation) lors des entretiens de conseil.

La description des traces verbales liées aux émotions de valence positive et des relations émotionnelles entre les personnes en interaction, prochaines étapes de notre recherche, pourrait conduire à deux investigations. La première concerne un hypothétique mécanisme de contagion émotionnelle. L'analyse de séries d'entretiens permet d'observer une diminution des émotions négatives au fil des rencontres. Ceci nous incite à en explorer les raisons : en particulier, le conseiller joue-t-il un rôle? Une première étude de type statistique suggère que ce dernier tend à exprimer des émotions de façon à amener la tonalité de l'interaction vers la neutralité. Lorsque l'état émotionnel d'un apprenant est positif, l'état corrélé du conseiller tend vers le neutre et lorsque l'état de l'apprenant est négatif, l'état corrélé du conseiller est positif. Si ce phénomène de contagion apparaissait explicatif des changements émotionnels, une deuxième investigation consisterait à identifier les façons dont le conseiller soutient l'état motivationnel de l'apprenant, et à en déterminer l'impact sur l'apprentissage, notamment en observant l'évolution de la méthodologie de l'apprenant.

De façon plus générale, ces investigations sont susceptibles de contribuer à une meilleure connaissance des fonctions des émotions, les hypothèses générées dans ce cadre pouvant en outre rendre intelligible une modélisation dynamique des relations émotionnelles dans l'entretien de conseil.

\section{RÉFÉRENCES BIBLIOGRAPHIQUES}

Abe, D., Henner Stanchina, C. \& Smith, P. (1975). New Approaches to Autonomy: Two Experiments in Self-Directed Learning. Mélanges Crapel $^{6}, 57-78$.

Bown, J., \& White, C. (2011). A Social and Cognitive Approach to Affect in SLA. International Review of Applied Linguistics in Language Teaching, 48 (4), 331-353.

Carette, E., \& Castillo, D. (2004). Devenir conseiller : quels changements pour l'enseignant? Mélanges Crapel, 27, 71-97.

6. Les Mélanges Crapel ne sont pas dotés de numéro de volume jusqu'en 1990. 
Carette, E., Guély, E. \& Pereiro, M. (2011). Création et accompagnement d'un centre de langues privé : un exemple de collaboration entre recherche en didactique des langues et entreprise. Mélanges Crapel, $32,25-43$.

Carney, D. R. \& Colvin, C. R. (2010). The Circumplex Structure of Affective Social Behaviour. Social Psychological and Personality Science, $1,73-80$.

Ciekanski, M. (2005). L'accompagnement à l'autoformation en langue étrangère : contribution à l'analyse des pratiques professionnelles. Étude des dimensions langagières et formatives des pratiques dites «de conseil» dans des systèmes d'apprentissage autodirigé en LE. Thèse de doctorat, Université Nancy 2, Nancy.

Ciekanski, M. (2007). Fostering Learner Autonomy: Power and Reciprocity in the Relationship between Language Learner and Language Learning Adviser. Cambridge Journal of Education, 37 (1), 111-127.

Cohen, A. S., Minor, K. S., Najolia, G. M. \& Hong, S. L. (2009). A Laboratory-Based Procedure for Measuring Emotional Expression from Natural Speech. Behavior Research Methods, 41 (1), 204-212.

Cosmides, L. \& Tobby, J. (2000). Evolutionary Psychology and the Emotions. Dans M. Lewis \& J. M. Haviland-Jones (dir.), Handbook of Emotions (p. 91-115). New York, NY : Guilford.

Cowie, R., Douglas-Cowie, E., Savvidou, S., McMahon, E., Sawey, M. \& SchröDER, M. (2000). 'FEELTRACE': An Instrument for Recording Perceived Emotion in Real Time. Dans R. Cowie, E. Douglas-Cowie, E. \& M. Schröder (dir.), Proceedings of the ISCA Workshop on Speech and Emotion: A Conceptual Framework for Research (p. 19-24). Belfast, Irlande : Textflow.

Dewaele, J.-M. (2011). Reflections on the Emotional and Psychological Aspects of Foreign Language Learning and Use. Anglistik: International Journal of English Studies, 22 (1), 23-42.

DöRnYEI, Z. (2005). The Psychology of the Language Learner: Individual Differences in Second Language Acquisition. Mahwah, NJ : Lawrence Erlbaum.

Ekman, P. (1999). Basic Emotion. Dans T. Dalgleish \& M. Power (dir.), Handbook of Cognition and Emotion (p. 45-60). Sussex, Angleterre : John Wiley \& Sons.

Forbes-Riley, K. \& Litman, L. (2004). Predicting Emotion in Spoken Dialogue from Multiple Knowledge Sources. Proceedings of HLT/ NAACL'2004, 201-208.

Gremmo, M.-J. (1995). Conseiller n'est pas enseigner : le rôle du conseiller dans l'entretien de conseil. Mélanges Crapel, 22, 35-61. 
Gremmo, M.-J. \& Holec, H. (1987). Évolution de l'autonomie de l'apprenant : le cas de l'apprenant D. Mélanges Crapel, 85-101.

Haverkate, H. (1994). La cortesía verbal. Estudio pragmalingüístico. Madrid, Espagne : Gredos.

Holec, H. (1990). Qu'est-ce qu'apprendre à apprendre? Mélanges Crapel, 75-87.

HuRd, S. (2008). Affect and Strategy Use in Independent Language Learning. Dans S. Hurd \& T. Lewis (dir.). Language Learning Strategies in Independent Settings (Second Language Acquisition) (p. 218-236). Bristol, Angleterre : Multilingual Matters.

KJisiK, F., Voller, P., Aoki, N. \& NaKata, Y. (2009). Mapping the Terrain of Learner Autonomy, Learning Environments, Learning Communities and Identities. Tampere, Finlande: Tampere University Press.

Lee, C. M. \& Narayanan, S. (2005). Towards Detecting Emotions in Spoken Dialogs. IEEE Transactions on Speech and Audio Processing, 13 (3), 293-303.

LeECH, G. (1983). Principles of Pragmatics. Londres, Angleterre: Longman. Méndez López, M. (2011). The Motivational Properties of Emotions in Foreign Language Learning. Colombian Applied Linguistics Journal, 2, 43-59.

NygaARd, L. C. \& QueEn, J. S. (2008). Communicating Emotion: Linking Affective Prosody and Word Meaning. Journal of Experimental Psychology. Human Perception and Performance, 34 (4), 1017-1030.

Pell, M. D., Jaywant, A., Monetta, L. \& Kotz, S. A. (2011). Emotional Speech Processing: Disentangling the Effects of Prosody and Semantic Cues. Cognition and Emotion, 25 (5), 834-853.

RiLEY, P. (1994). Aspects of Learner Discourse: Why Listening to Learners is so Important. Dans E. Esch (dir.), Self-Access and the Adult Learner (p. 7-18). Londres, Angleterre : CILT.

Riley, P. \& Gremmo, M.-J. (1997). Autonomie et apprentissage autodirigé : l'histoire d'une idée. Mélanges Crapel, 23, 81-107.

Russell, J. A. (1980). A Circumplex Model of Affect. Journal of Personality and Social Psychology, 39, 1161-1178.

SCHERER, K. R. (1989). Vocal Correlates of Emotion. Dans A. Manstead \& H. Wagner (dir.). Handbook of Psychophysiology: Emotion and Social Behaviour (p. 165-197). Chichester, Angleterre : Wiley.

Scherer, K. R. (2005). What are Emotions? And How Can They Be Measured? Social Science Information, 44 (4), 695-729.

Scherer, K. R., Banse, R. \& Wallbott, H. G. (2001). Emotion Inferences from Vocal Expression Correlate Across Languages and Cultures. Journal of Cross-Cultural Psychology, 32 (1), 76-92. 